\title{
Kidney Regeneration in Fish
}

\author{
Alan J. Davidson \\ Department of Molecular Medicine and Pathology, School of Medical Sciences, Faculty of Medical and \\ Health Sciences, The University of Auckland, Auckland, New Zealand
}

\section{Key Words}

Nephron · Regeneration · Fish

\begin{abstract}
Background: Chronic and acute kidney injury damages nephrons, the blood filtering tubules in the kidney. Although mammalian kidneys can regenerate the tubular epithelium of the nephron, no new nephrons are made during adulthood. In contrast, fish are capable of growing nephrons de novo throughout their life. A better understanding of this 'neo-nephrogenic' response in fish may lead to the development of novel regenerative therapies to treat kidney disease in humans. Summary: In this review, nephron formation in the fish mesonephric kidney during normal growth and in response to acute injury is examined at the morphological and molecular levels. Included is an overview of the recent discovery of migratory nephron progenitors that, following transplantation, can engraft host kidneys and give rise to functional nephrons. Key Messages: Mesonephric nephron progenitors appear during the larval stage, migrate together to form clusters, activate the expression of conserved nephrogenic genes, and epithelialize into nascent nephrons in a process that resembles mammalian nephron formation. Nephron progenitors persist in the adult fish kidney and continue to add new nephrons at a basal rate as the fish grows in size. Following acute kidney injury, nephron formation is
\end{abstract}

significantly increased, allowing the fish to rapidly regenerate lost nephrons. Transplantation of nephron progenitors into the kidney results in the formation of donor-derived nephrons in the recipient fish.

(c) 2014 S. Karger AG, Basel

\section{Introduction}

Regeneration is a process by which damaged or lost cells are replaced in order to restore the structure and function of a tissue. The regenerative potential of organs varies within an organism and can also show marked differences between species. A notable example of this is the vertebrate kidney, an organ comprised of blood-filtering tubules called nephrons that regulate waste excretion and salt and water homeostasis. In mammals, the renal tubules can self-repair in response to injury [1]. In fish, this repair pathway is also likely operative; however, the fish kidney has the additional capacity to generate entire nephrons de novo [2-6]. Recent studies in zebrafish suggest that this 'neo-nephrogenic' process recapitulates conserved pathways utilized during vertebrate kidney organogenesis [6]. Here, we review fish nephron formation and our current understanding of fish renal regeneration in response to injury.

\section{KARGER}

E-Mail karger@karger.com

www.karger.com/nee (c) 2014 S. Karger AG, Basel

$1660-2129 / 14 / 1262-0045 \$ 39.50 / 0$
Alan J. Davidson

Department of Molecular Medicine and Pathology, School of Medical Sciences Faculty of Medical and Health Sciences, The University of Auckland

Private Bag 92019, Auckland 1142 (New Zealand)

E-Mail a.davidson@ auckland.ac.nz 


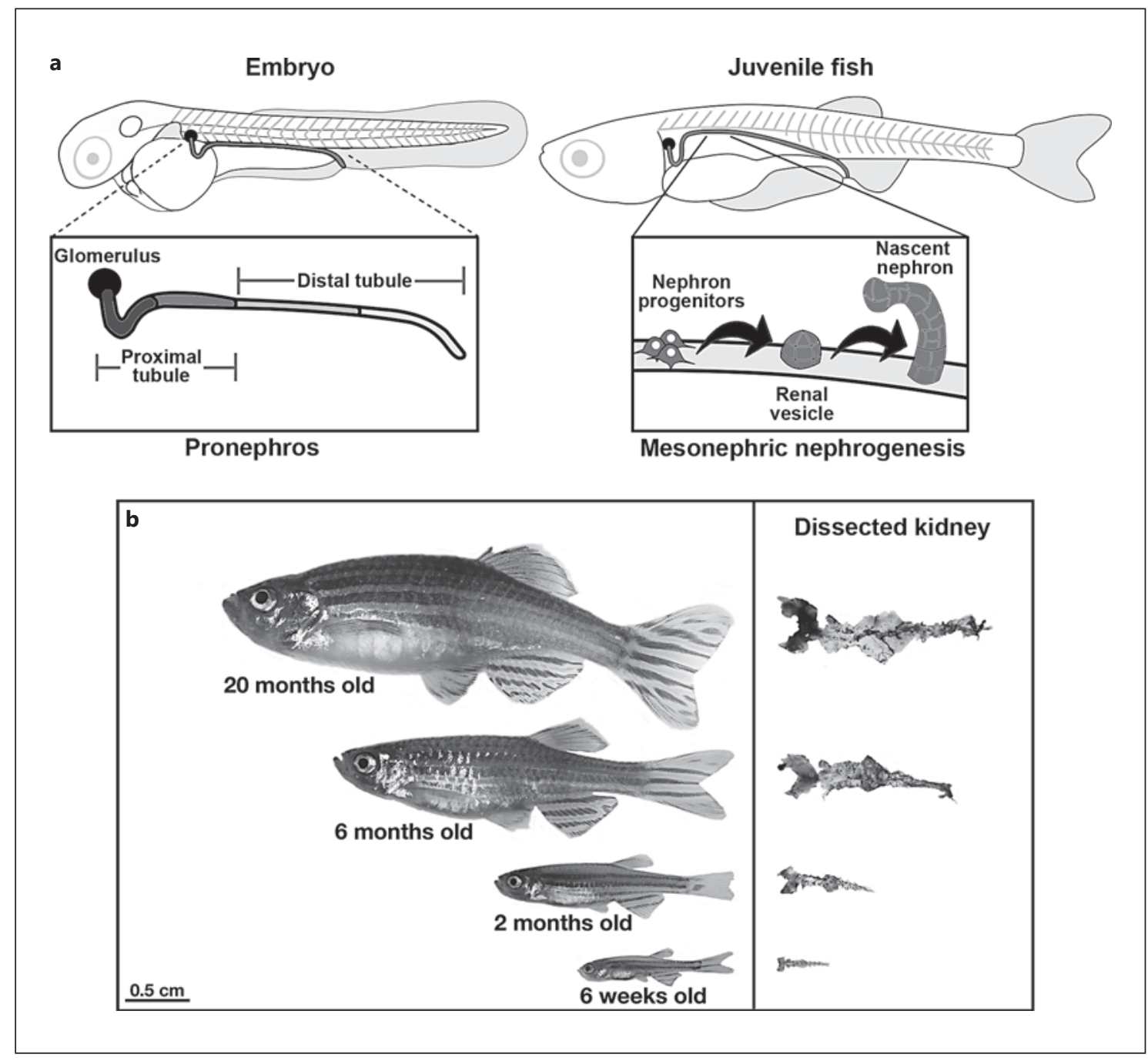

Fig. 1. Overview of the growth and structure of zebrafish nephrons. a Schematic representations of embryonic and juvenile zebrafish showing the segmental arrangement of the pronephric tubule (left) and the formation of mesonephric nephrons from migratory nephron progenitors (right). b Images of zebrafish and their respective kidneys at various ages showing how kidney mass increases with body size.

\section{Fish Kidney Formation}

The vertebrate kidney is unique among the organs in that 2 or 3 kidney types arise sequentially during early life. In general, fish form the first 2 kidneys, called the pronephros and mesonephros, with the latter being the permanent adult kidney (mammals develop a third kidney, the metanephros, as their final adult kidney). In fish, the pronephric kidney plays a vital role in osmoregulation, as embryos usually develop externally and are therefore exposed to environmental pressures on water and salt homeostasis. For freshwater fish such as zebrafish, the pro- nephros acts to reclaim solutes and void excess water and remains functional for many weeks following fertilization [7]. Structurally, the zebrafish pronephros is relatively simple and comprises 2 fused bilateral nephrons (sharing a common glomerulus) with tubules divided into 2 proximal segments and 2 distal segments [8] (fig. 1a). The pronephric nephrons derive from bilateral stripes of intermediate mesoderm cells that express renal regulators such as orthologues of the transcription factors Pax2, Lhx1, and WT1 [7]. The intermediate mesoderm differentiates in situ into renal epithelial cells and is concomitantly 'patterned' along the proximo-distal axis into the cells of the 
glomerular blood filter and the proximal and distal tubules. Key regulators of this process include retinoic acid signaling and the HNF1- $\beta$ transcription factor $[8,9]$.

The mesonephric kidney forms in juvenile fish $(2-3$ weeks after fertilization) and is made up of hundreds of nephrons that branch distally $[5,6]$. In zebrafish, the mesonephric nephrons show a segmentation pattern similar to that of the pronephros, comprising 2 proximal segments (PI and PII) and 2 distal segments (distal early and distal late). Early histological studies in the Sandkhol carp, a tropical freshwater fish, suggested that mesonephric nephrons arise from clusters of nephrogenic cells that form on top of the bilateral pronephric tubules [10]. Consistent with this, live imaging of transgenic zebrafish larvae, in which green fluorescent protein (GFP) is expressed in nephrogenic cells under the control of the lhxla promoter, showed that migratory cells first appear on the pronephric tubules and then coalesce, epithelialize, and elongate into new nephrons that fuse with the pronephros (fig. 1a) [6].

Unlike the mammalian metanephric kidney, where nephrogenesis ceases around birth, the fish mesonephric kidney continues to add new nephrons as the body mass increases (fig. 1b). This relationship between nephron number and fish size was documented over 80 years ago in goldfish, where a $\sim 10$-fold difference in body weight is associated with a $\sim 2.5$-fold increase in nephron number [11]. This direct relationship between nephron number and size presumably reflects the need for increased kidney function as the fish's body mass and fluid volume increase with growth. In mammals, this problem is solved during postnatal growth by increasing nephron performance via increases in size and the glomerular filtration rate rather than by the addition of new nephrons [12]. The reason why fish and mammals have adopted these different strategies is not known. Speculative possibilities include physiological differences such as constraints imposed by low blood pressure in fish versus high blood pressure in mammals (giving mammals a greater dynamic range in glomerular filtration pressure) or structural differences in nephron organization, such as the presence of the loop of Henle in mammals [for more details, see 13 and 14].

\section{Adult Renal Regeneration in Fish}

While fish add new nephrons at a basal rate as they grow, neo-nephrogenesis can be significantly increased if the kidney is acutely damaged. Pioneering work in gold- fish using intraperitoneal injections of the nephrotoxic agent hexachlorobutadiene showed that proximal tubule injury induces significant neo-nephrogenesis within 2 weeks of exposure [2]. The source of these nephrons was traced to proliferating cell clusters, like those described in Sandkhol carp larvae, which formed on the distal nephron. Similar findings were reproduced more recently in zebrafish using gentamicin to induce proximal tubule necrosis $[5,6]$. While examining $l h x 1 a-G F P$ transgenic zebrafish, fluorescent cells were found scattered throughout the adult kidney as single cells, clusters, and immature nephron outgrowths [6]. Transplantation of purified lhxla-GFP+ clusters, comprising 10-30 cells, directly into the kidneys of immunocompromised recipient fish resulted in the formation of functional donor-derived nephrons near the site of injection, confirming that the clusters contain nephron progenitors. Interestingly, donor-derived nephrons were also found in more distant sites, consistent with the nephron progenitors being highly migratory [6]. The mobile nature of these cells is unexpected as nephron progenitors are generally considered to be localized to an anatomically defined niche [15]. Transplantation experiments in which kidney cell suspensions carrying red or green fluorescent reporters were mixed and then injected into recipient fish resulted in the formation of chimeric red- and green-labeled nephrons, providing further proof that the lhxla-GFP+ nephrogenic clusters arise from the coalescence of individual migrating cells [6].

The signals that recruit $\operatorname{lh} x 1 a-\mathrm{GFP}+$ cells to the distal nephron to form nephrogenic clusters are not known. Nephrectomy experiments in the little skate have demonstrated that loss of nephrons from one kidney leads to increased nephrogenesis in the contralateral kidney, suggestive of a systemic regulatory mechanism [3]. One possibility is that fish kidneys have a way of sensing systemic changes in salt or water homeostasis. In support of this, pink salmon raised in freshwater (where voiding water and reclaiming salts are critical to the maintenance of ionic balance) develop significantly more nephrons than fish raised in seawater [16]. A potential mediator of this response is the hormone prolactin, which is released systemically from the pituitary and in fish plays a major role in osmoregulation [17]. Injection of prolactin into eels transferred to seawater induces neo-nephrogenesis, mimicking a response they would normally only have in freshwater [18]. Whether prolactin acts directly to induce neonephrogenesis or indirectly via changes in water or salt homeostasis is not known. The advantage of this kind of systemic sensing mechanism is that it permits nephrons 
to be added in response to the demands placed on kidney function rather than being tied to a general growth pathway such as the growth hormone-IGF axis.

\section{Molecular Regulation of Mesonephric Nephrogenesis}

Morphologically, the process of fish neo-nephrogenesis appears to closely follow what is observed in the metanephric kidney where cap mesenchyme cells, a pool of self-renewing nephron progenitors, give rise to pretubular aggregates that epithelialize into a ball called a renal vesicle and then elongate into a nephron. It is not yet clear whether lhxla-GFP+ cells are equivalent to cap mesenchyme cells or pretubular aggregates or they correspond to an entirely new type of nephron progenitor cell. In mammals, $L h x 1$ is expressed by pretubular aggregates, raising the possibility that $l h x 1 a-G F P+$ cells represent a migratory form of these cells [19]. However, a microarray analysis comparing lhxla-GFP+ cells to mouse Six $2+$ cells (comprising cap mesenchyme and pretubular aggregates) did not reveal significant similarities, although the lhx1a-GFP+ cells did express Six2 and WT1 [6]. By contrast, once the lhxla-GFP+ clusters epithelialize into renal vesicles, they upregulate a number of established nephrogenic factors including orthologues of $\mathrm{Pax} 2, \mathrm{Pax} 8$, WT1, and FGF8 [6]. The activation of these genes, which are implicated in embryonic kidney formation in many vertebrate species, suggests that a conserved program of nephrogenesis is initiated in $\operatorname{lh} x 1 a-\mathrm{GFP}+$ clusters.

As early as the renal vesicle stage, there is molecular evidence for cell fate patterning, with $w t 1 b$ and $l h x 1 a$ showing polarized expressions in presumptive proximal and distal regions of the nascent nephron [6]. Similar observations have been documented during mammalian metanephric development, further reinforcing the notion that the mechanisms of nephrogenesis are conserved between fish and mammals [20]. The expression of lhxla remains localized to the distal-most cells of the developing nephron at the point of fusion with the underlying tubule. The cells at this junction appear to insert themselves into the underlying tubule, ultimately resulting in the joining of the lumen of the nascent nephron with the existing tubule [6]. The Lhx1 transcription factor has been implicated as a regulator of cell movement, raising the possibility that it may play a role in the nephron fusion process [21].

\section{Conclusions}

Studies to date suggest that fish mesonephric nephron progenitors appear during the larval stage and coalesce into clusters on top of the pronephric tubules. The cells in these clusters activate the expression of renal regulators and epithelialize into nascent nephrons that fuse with the underlying tubule. This process resembles mammalian nephrogenesis, both morphologically and at the molecular level, suggesting that the pathways operating in fish are conserved in humans. However, unlike in mammals, fish nephron progenitors persist in the adult kidney and continue to add new nephrons as the fish grows, presumably to ensure that kidney function keeps pace with increases in body mass. The signals regulating the rate of this neonephrogenesis are not well characterized but may be linked to the ability of the fish to sense changes in salt or fluid balance. Following acute kidney injury, neo-nephrogenesis increases dramatically allowing fish to rapidly regenerate lost nephrons. A better understanding of the fish neo-nephrogenic response may lead to new avenues to explore in the quest to develop renal regeneration therapies in patients with chronic and acute kidney injury.

\section{References}

$\checkmark 1$ Humphreys BD, Valerius MT, Kobayashi A, Mugford JW, Soeung S, Duffield JS, McMahon AP, Bonventre JV: Intrinsic epithelial cells repair the kidney after injury. Cell Stem Cell 2008;2:284-291.

2 Reimschuessel R, Bennett RO, May EB, Lipsky MM: Development of newly formed nephrons in the goldfish kidney following hexachlorobutadiene-induced nephrotoxicity. Toxicol Pathol 1990;18:32-38.
-3 Elger M, Hentschel H, Litteral J, Wellner M, Kirsch T, Luft FC, Haller H: Nephrogenesis is induced by partial nephrectomy in the elasmobranch Leucoraja erinacea. J Am Soc Nephrol 2003;14:1506-1518.

4 Watanabe N, Kato M, Suzuki N, Inoue C, Fedorova S, Hashimoto H, Maruyama S, Matsuo S, Wakamatsu Y: Kidney regeneration through nephron neogenesis in medaka. Dev Growth Differ 2009;51:135-143.
5 Zhou W, Boucher RC, Bollig F, Englert C, Hildebrandt F: Characterization of mesonephric development and regeneration using transgenic zebrafish. Am J Physiol Renal Physiol 2010;299:F1040-F1047.

-6 Diep CQ, Ma D, Deo RC, Holm TM, Naylor RW, Arora N, Wingert RA, Bollig F, Djordjevic G, Lichman B, Zhu H, Ikenaga T, Ono F, Englert C, Cowan CA, Hukriede NA, Handin RI, Davidson AJ: Identification of adult nephron progenitors capable of kidney regeneration in zebrafish. Nature 2011;470:95100.
48 
7 Drummond IA, Davidson AJ: Zebrafish kidney development. Methods Cell Biol 2010; 100:233-260.

8 Wingert RA, Selleck R, Yu J, Song HD, Chen Z, Song A, Zhou Y, Thisse B, Thisse C, McMahon AP, Davidson AJ: The cdx genes and retinoic acid control the positioning and segmentation of the zebrafish pronephros. PLoS Genet 2007;3:1922-1938.

\9 Naylor RW, Przepiorski A, Ren Q, Yu J, Davidson AJ: HNF1 $\beta$ is essential for nephron segmentation during nephrogenesis. J Am Soc Nephrol 2013;24:77-87.

10 Moghe MA: Development of the mesonephros in a teleostean - Thynnichthys sandkhol. Quart J Micro Sci 1945;s2-85:129-151.

11 Nash J: The number and size of glomeruli in kidneys of fishes, with observations on the morphology of the renal tubules of fishes. Am J Anat 1931;47:425-445.
12 McCrory WW: Renal function in the postnatal period; in Wallace W, McCrory MD (eds): Developmental Nephrology. Cambridge, Harvard University Press, 1972, pp 123-161.

13 Davidson AJ: Uncharted waters: nephrogenesis and renal regeneration in fish and mammals. Pediatr Nephrol 2011;26:1435-1443.

14 Romagnani P, Lasagni L, Remuzzi G: Renal progenitors: an evolutionary conserved strategy for kidney regeneration. Nat Rev Nephrol 2013;9:137-146.

15 Carroll TJ, Das A: Defining the signals that constitute the nephron progenitor niche. J Am Soc Nephrol 2013;24:873-876.

16 Ford P: Studies on the development of the kidney of the Pacific pink salmon [Oncorhynchus gorbuscha (Walbaum)]. 2. Variation in glomerular count of the kidney of the pacific pink salmon. Can J Zool 1958;36:45.

17 Sakamoto T, McCormick SD: Prolactin and growth hormone in fish osmoregulation. Gen Comp Endocrinol 2006; 147:24-23.

18 Olivereau M, Olivereau J: Effects of hypophysectomy and prolactin replacement on eel kidney structure during adaptation to sea water. Acta Zool 1977;58:103-115.
19 Kobayashi A, Kwan KM, Carroll TJ, McMahon AP, Mendelsohn CL, Behringer RR: Distinct and sequential tissue-specific activities of the LIM-class homeobox gene Lim 1 for tubular morphogenesis during kidney development. Development 2005;132:2809-2823.

20 Georgas K, Rumballe B, Valerius MT, Chiu HS, Thiagarajan RD, Lesieur E, Aronow BJ, Brunskill EW, Combes AN, Tang D, Taylor D, Grimmond SM, Potter SS, McMahon AP, Little MH: Analysis of early nephron patterning reveals a role for distal RV proliferation in fusion to the ureteric tip via a cap mesenchyme-derived connecting segment. Dev Biol 2009;332:273-286.

21 Hukriede NA, Tsang TE, Habas R, Khoo PL, Steiner K, Weeks DL, Tam PP, Dawid IB: Conserved requirement of Lim 1 function for cell movements during gastrulation. Dev Cell 2003;4:83-94. 